\section{Internações no Sistema de Serviços Hospitalares, SUS e não SUS: Brasil, 2006}

\section{Hospital admissions in the hospital health care system, SUS and non- SUS: Brazil, 2006}

\section{Marizélia Leão Moreira'}

\section{Hillegonda Maria DutilhNovaes"}

'Agência Nacional de Saúde Suplementar - Rio de Janeiro (RJ), Brasil

"Departamento de Medicina Preventiva da Faculdade de Medicina da Universidade de São Paulo (USP) - São Paulo (SP), Brasil

Trabalho realizado na Faculdade de Medicina da Universidade de São Paulo (USP) - São Paulo (SP), Brasil. Fonte de financiamento: nenhuma.

Correspondência: Marizélia Leão Moreira - R. Dias Ferreira, 655, apto 501 - Leblon - CEP: 22431-050 Rio de Janeiro (RJ), Brasil - E-mail: marizelialm@yahoo.com.br

Conflito de interesses: nada a declarar.

\section{Resumo}

O objetivo deste estudo foi descrever as admissões, SUS e não SUS, no Sistema de Serviços Hospitalares no Brasil no ano de 2006, adotando o indivíduo internado como unidade de análise. As fontes dos dados foram o Sistema de Informação Hospitalar (SIH/SUS) e de Comunicação de Internação Hospitalar (CIH) referentes a 2006 e 2007. A identificação do indivíduo foi feita pelo método probabilístico de associações de registros (linkage) e para a composição dos dados da internação aplicaram-se algoritmos de composição nos registros de cobranças e de comunicações das internações. Foram analisadas 12.391.990 internações com ao menos um dia de duração no ano de 2006. A natureza do método probabilístico que encerra certo grau de imprecisão, a adoção de parâmetros conservadores a fim de evitar a inclusão de falsos positivos, tanto quanto a subnotificação da CIH representam as possíveis limitações do estudo. $\mathrm{O}$ Sistema de Serviços Hospitalares no Brasil apresentou taxa de hospitalização de $5,6 \%$, sendo $5,0 \%$ SUS e 1,6\% não SUS, diferenciando-se segundo Unidade da Federação de ocorrência. Os dados das internações não financiadas pelo SUS coletados pela CIH agregam informações importantes para a análise da assistência hospitalar no país. Os dados administrativos do SUS são válidos para análises de internações $\mathrm{e}$ os algoritmos de composição dos dados de internação, a partir da cobrança, aprimoram a análise do Sistema de Serviços Hospitalares no Brasil.

Palavras-chave: sistemas de informação hospitalar; hospitalização; sistema único de saúde; epidemiologia. 


\section{Abstract}

The objective of this study was to analyze admissions in the hospital health care system in Brazil in the year of 2006, starting from the admitted subjects. The initial data were the admissions SUS (SIH), non-SUS (CIH) in 2006 and 2007. The identification of the individual was by probabilistic method of associations of registrations (linkage) and, for the composition of the admission data, specific algorithms were applied to the data. A total of 12,391,990 admissions with at least one day in hospital in the year of 2006 were analyzed. The imprecise nature of the probabilistic method at a certain degree, the adoption of conservative parameters in order to avoid the inclusion of false positive, and the subnotification of $\mathrm{CIH}$ represent possible limitations of this study. The Hospital Services System in Brazil presented admission rate of $5.6 \%$ in total, $5.0 \%$ SUS and $1.6 \%$ non-SUS, with differences according to age and region. The non-SUS admissions data collected by $\mathrm{CIH}$ give important additional information to the analysis of the hospital utilization in the country. The SUS administrative data are valid for analyses of admissions and the algorithms for admission data composition enhance the analysis of the Hospital Services System in Brazil.

Keywords: hospital information systems; hospitalization; unified health system; epidemiology.

\section{Introdução}

A análise do perfil de utilização de serviços de saúde em geral é complexa, ao resultar da articulação entre oferta e acesso aos serviços de saúde e das características sociodemográficas e epidemiológicas dos usuários $^{1,2}$. As alterações do perfil demográfico e epidemiológico da população, as transformações na organização da assistência e a contínua incorporação e demanda por novas tecnologias implicam necessidade de estudos cada vez mais detalhados da assistência hospitalar no país e a criação de instrumentos que apoiem a gestão do Sistema Único de Saúde (SUS) e do Sistema de Assistência Suplementar (não SUS) ${ }^{3-9}$.

Estudo recente, com os dados do SUS, mostrou que o volume de internações por esta fonte de financiamento vem diminuindo. A redução observada foi maior na assistência obstétrica e na clínica médica, em especial nos serviços privados lucrativos $^{6}$. Entretanto, outros estudos, baseados nos dados da Pesquisa Nacional por Amostra de Domicílios (PNAD), apontam aumento da participação do SUS no volume das internações referidas ${ }^{7,8}$ com tendência para diminuição nos tempos de permanência $^{7}$, e padrão semelhante na busca por serviços dos usuários SUS e dos não SUS .

No Brasil, o SUS tem como princípios o acesso universal aos serviços e atenção integral à saúde. No entanto, os resultados da PNAD de 2008 apontaram a população residente coberta por planos de saúde como sendo de $25,9 \%$ no país com diferenciação entre as regiões: $13,3 \%$ na Norte, $13,2 \%$ na Nordeste, $24,6 \%$ na Centro-Oeste, $35,6 \%$ na Sudeste e $30,0 \%$ na Sul ${ }^{10}$. A significativa participação de outras fontes de financiamento das internações além do SUS impõe a necessidade da ampliação das informações a serem analisadas, para que seja possível conhecer a assistência hospitalar prestada aos residentes no país.

A Comunicação de Internação Hospitalar (CIH), implantada em julho de 1999, é o instrumento que coleta os dados de internação financiadas pelo Sistema de Assistência 
Suplementar de todos os estabelecimentos de saúde situados em território nacional, integrantes ou não do SUS. Sendo assim, a CIH é o sistema de coleta dos dados das internações não SUS, aquelas financiadas por planos de saúde, por particulares, pela própria unidade de internação (gratuito), as com recursos próprios das Secretarias Municipais de Saúde e Secretarias Estaduais de Saúde e Seguro para Danos Pessoais Causados por Veículos Automotores em Vias Terrestres $(\text { DPVAT) })^{11}$.

Há publicações que analisam internações pelo SUS, tendo por base o Sistema de Informações Hospitalares (SIH/SUS), em diferentes perspectivas ${ }^{3,12-15}$. Todavia, não foram encontradas publicações sobre as internações não SUS, com base na CIH. Existem estudos sobre internações hospitalares, independente da fonte de financiamento, com dados do inquérito populacional da PNAD, baseada em utilização referida pelos usuários. Estudo com dados da última PNAD (2008) divulgou um coeficiente geral de internação hospitalar nacional de 7,1\%. Das internações referidas pelos indivíduos no período de 12 meses, 24,7\% foram informadas como tendo sido financiadas por meio de plano de saúde ${ }^{11}$.

Os sistemas de informação nacionais que registram dados administrativos relativos aos sistemas de saúde atendem às necessidades específicas da gestão, não tendo sido planejados para subsidiar pesquisas. Contudo no Brasil, o SIH conquanto implantado com a finalidade de pagamento é frequentemente usado em estudos sobre assistência hospitalar ${ }^{3,12-19}$. O objetivo desse artigo foi descrever as admissões, SUS e não SUS, no Sistema de Serviços Hospitalares no Brasil no ano de 2006, adotando o indivíduo internado como unidade de análise.

\section{Métodos}

Foram analisadas as internações pagas pelo SUS e as não SUS, informadas ao Ministério da Saúde, com ao menos um dia de ocorrência em 2006. As fontes de dados foram: a) o Cadastro Nacional de
Estabelecimentos de Saúde (CNES) utilizado nesta pesquisa para caracterizar o hospital no qual ocorreram as internações; b) o Sistema de Informações Hospitalares (SIH) para as internações SUS; c) a Comunicação de Internações Hospitalares (CIH) para as internações não SUS. Os dados SUS e não SUS analisados referem-se ao período de competência de janeiro/2006 a dezembro/2007, processados até julho/2008 e outubro/2008, respectivamente, em razão do fluxo da coleta dos dados e a fim de incluir internações de 2006, cujos dados foram enviados em 2007. Os dados foram fornecidos pelo Datasus em arquivos no formato data base file (DBF), mediante a apresentação da declaração de aprovação do projeto de pesquisa pela Comissão de Ética para Análises de Projetos de Pesquisas CAPPesq da Diretoria Clínica do Hospital das Clínicas e da Faculdade de Medicina da Universidade de São Paulo.

Com os dados de 2006 e 2007, que totalizaram 27.224.577 registros, foi organizada uma base de dados para localizar o indivíduo internado e as suas internações. Durante a organização dessa base, os registros inconsistentes em ao menos uma das seguintes variáveis: nome, data de nascimento e sexo, foram excluídos do processo de vinculação. A localização e atribuição de um código para o indivíduo foram por associação de registros, utilizando-se o aplicativo Reclink ${ }^{20,21}$ e as variáveis nome, sexo, município de residência e data de nascimento. Para o campo "nome" foi estabelecido o algoritmo de comparação "aproximado" com os parâmetros: sensibilidade=99, 1-especificidade e limiar de concordância=85. Já para o campo "data de nascimento" adotou-se o algoritmo de comparação "exato". O escore mínimo foi definido em 9,6. A associação de registros para a codificação da identificação do indivíduo foi por Unidade da Federação (UF) de ocorrência, uma vez que se observou ser pequeno o fluxo de atendimento entre as UF.

Em seguida, codificou-se de forma unívoca, também através do Reclink, o evento: 
registro de cobrança e de comunicação de internação, a fim de rastrear a duplicidade de registros. O evento foi definido pelo conjunto de dados composto pelo código do hospital segundo o CNES, o código do indivíduo, a data de internação, a data de saída e o diagnóstico principal, sendo todos comparados pelo algoritmo "exato". O escore mínimo de aceitação foi igual a 13,0. Como no SIH o evento pode corresponder a uma cobrança parcial, de parte do período ou de alguns procedimentos da internação, situação essa também aceita nas CIH do período pesquisado, foram definidos três algoritmos para identificar os eventos de uma mesma internação e compor o registro de seus dados. Os algoritmos basearam-se no CNES do hospital, no código do indivíduo, atribuído pelo Reclink, nas datas de entrada e de saída, informados na cobrança. O primeiro algoritmo identificou os atendimentos com apenas uma cobrança por motivo de alta, transferência ou óbito. $\mathrm{O}$ segundo identificou os atendimentos com uma ou mais cobranças intermediárias, aqueles cujo motivo de cobrança foram permanência, alta administrativa ou para reoperação. Todos com o intervalo menor ou igual a um dia, em relação à maior data de saída anterior, e que possuíam também uma cobrança final (alta, transferência ou óbito). Já o terceiro buscou os atendimentos com cobranças intermediárias, ou seja, os que não possuíam uma cobrança por alta, transferência ou óbito. Com a aplicação dos algoritmos puderam-se localizar todos os eventos de um mesmo atendimento e foi possível compor o registro da internação completa. O atendimento recebeu um código unívoco com base no CNES do hospital, código do indivíduo e do evento. E neste, os dados do indivíduo relativos ao sexo, à data de nascimento e ao município de residência, basearam-se no primeiro evento de cobrança. Para os campos de procedimento realizado, diagnóstico principal e secundário, motivo de saída, idade e ocorrência de óbito foram utilizados os dados da última cobrança. Os dias de permanência e as diárias de UTI foram obtidos pela soma dos valores registrados no conjunto de cobranças que compôs o atendimento. Dentre as internações, definidas como o atendimento com permanência acima de 24 horas, foram identificadas as de 2006, aquelas cujo período de ocorrência abrangeu ao menos um dia entre 01/01/2006 e 31/12/2006, independentemente desta ser a data de início ou final da internação.

Foram calculadas as taxas de internação pelo método usualmente utilizado, baseado no número médio de internações financiadas pelo SUS ${ }^{22}$, e pelo número de indivíduos internados, obtidos com base nos processos de linkage desenvolvidos. A taxa de internação foi padronizada com método direto, nas seguintes faixas etárias da população: menor de 1 ano, 1 a 4 anos, 5 a 9 anos, 10 a 19 anos, 20 a 59 anos e 60 anos e mais.

\section{Resultados}

Foram contabilizadas 12.391 .990 internações completas e 10.545.249 indivíduos internados (Tabela 1). Foram identificadas diferenças quanto à distribuição etária das internações em relação à população, com mais indivíduos internados nas faixas etárias de menores de 1 ano e de 60 anos e mais (Tabela 1). Com relação à distribuição das internações por sexo, verificou-se maior proporção de mulheres $(60,8 \%)$, cujo percentual é superior à proporção do sexo feminino na população do país.

Nos óbitos hospitalares em 2006 (Tabela 1), em que o universo de análise está constituído pelos indivíduos e não pelas internações, a distribuição proporcional dos indivíduos internados e dos óbitos apresenta diferenças nas categorias analisadas. Os indivíduos de 60 anos e mais apresentaram a maior diferença em relação à proporção do mesmo grupo dentre os indivíduos internados $(60,9 \%$ dos pacientes que foram a óbito $v s 18,2 \%$ dos que foram internados). Quanto à distribuição por sexo, observa-se predomínio de óbitos hospitalares entre as pessoas do sexo masculino diferentemente do observado na distribuição de sexo dos pacientes internados. 
Tabela 1. Proporção de internações, indivíduos e óbito hospitalar por faixa etária e sexo. Brasil, 2006

Table 1. Proportion of admissions, individuals and hospital deaths by age group and sex. Brazil, 2006

\begin{tabular}{|c|c|c|c|c|c|}
\hline Faixa etária e sexo & $\begin{array}{c}\text { População } \\
(n=186.770 .613) \% \\
\end{array}$ & $\begin{array}{c}\text { Internações } \\
(\mathrm{n}=12.391 .990) \%\end{array}$ & $\begin{array}{c}\text { Indivíduos } \\
(\mathrm{n}=10.545 .249) \% \\
\end{array}$ & $\begin{array}{c}\text { População } \\
\text { internada \% }\end{array}$ & $\begin{array}{l}\text { Óbito hospitalar } \\
(\mathrm{n}=357.994) \%\end{array}$ \\
\hline TOTAL & & & & 5,6 & \\
\hline \multicolumn{6}{|l|}{ Faixa etária } \\
\hline$<1$ ano & 1,9 & 5,4 & 5,7 & 16,9 & 5,6 \\
\hline 1 a 4 anos & 7,8 & 6,8 & 7,0 & 5,1 & 0,9 \\
\hline 5 a 9 anos & 9,8 & 3,8 & 4,0 & 2,3 & 0,5 \\
\hline 10 a 19 anos & 20,8 & 10,8 & 11,5 & 3,1 & 1,5 \\
\hline 20 a 59 anos & 51,3 & 52,6 & 53,5 & 5,9 & 30,7 \\
\hline 60 anos e mais & 8,4 & 20,6 & 18,2 & 12,2 & 60,9 \\
\hline \multicolumn{6}{|l|}{ Sexo } \\
\hline Masculino & 49,2 & 39,2 & 37,6 & 4,3 & 54,2 \\
\hline Feminino & 50,8 & 60,8 & 62,4 & 6,9 & 45,8 \\
\hline
\end{tabular}

Fonte: MS/Datasus - Sistema de Informação Hospitalar (SIH) e Comunicação de Internação Hospitalar (CIH) competências 2006 e 2007. Source: MS/Datasus - Hospital Information System (HIS) and Communication Hospital (CIH) 2006 and 2007.

A Tabela 2 apresenta as taxas de internação por 100 habitantes segundo regiões e UF, calculadas segundo diferentes métodos. Com a aplicação do método indicado pela Rede Interagencial de Informações para a Saúde (RIPSA) $)^{22}$, obteve-se que 6,1 por 100 habitantes do país teriam sido internados pelo SUS (Tabela 2). Com a identificação dos indivíduos internados, obteve-se a taxa de 5,0 por 100 habitantes com internação pelo SUS, uma redução de $18 \%$ em relação ao cálculo anterior. Ao se incluir os dados disponíveis referentes às internações não SUS, a taxa passou a $5,6 \%$ por 100 habitantes com internação no Sistema de Serviços Hospitalares em 2006.

O método empregado para o cálculo das taxas (indivíduos $v$ s internações) para o SUS, teve maior impacto nos estados das regiões Sul e Sudeste, com as maiores reduções no Rio Grande do Sul (redução de 33,33\%), São Paulo (31,0\%), Paraná $(29,6 \%)$ e Santa Catarina (28,6\%) (Tabela 2). Quando agregados os dados disponíveis para as internações não SUS, o maior aumento em relação ao cálculo da taxa de indivíduos internados pelo SUS foi observado no estado de São Paulo (28,8\%), seguido pelo Rio Grande do Sul (21,5\%) e Santa Catarina (18,3\%). A taxa bruta de internação por 100 habitantes em 2006 apresentou, por região, valores entre $5,4 \%$ (Sudeste) e $6,2 \%$ (Centro-Oeste e Sul) e por UF, valores entre 4,0 (Rio de Janeiro) e $6,9 \%$ (Acre). Ao se adotar a taxa padronizada pela faixa etária da população, não se observou alteração na ordenação em que se colocam os valores segundo o estado da Federação (Figura 1).

Quanto ao total de internações, a participação do SUS foi de 87,9\%, do não SUS foi de $12,1 \%$ e $4,2 \%$ das internações no país utilizaram unidade terapia intensiva (UTI) (Tabelas 3 e 4). Dentre as internações que utilizaram UTI, observa-se que a faixa acima de 60 anos foi a mais frequente $(39,4 \%)$, seguida pelas faixas etárias entre 20 a 59 anos (35,8\%), e os menores de 1 ano de idade (16,5\%); o menor uso foi na faixa etária de 1 a 19 anos. Ao se analisar a distribuição das faixas etárias entre as internações que usaram UTI por fonte de financiamento, observa-se uma proporção mais elevada de internações em menores de 1 ano no SUS do que no não SUS e uma proporção maior de internações de paciente com 60 anos e mais no não SUS. As internações de indivíduos do sexo masculino predominam quanto ao uso da UTI nas duas modalidades de financiamento. Quanto aos motivos de saída do hospital, nas internações com uso de UTI, $69,4 \%$ tiveram alta, $4,5 \%$ foram transferidos e $22,6 \%$ foram a óbito. Observa-se que em apenas $0,1 \%$ dessas internações, foi registrada a 
Tabela 2. População e taxa de internação, com diferentes métodos de cálculo, por região e Unidade da Federação de residência. Brasil, 2006

Table 2. Population and admission rates with different methods, for region and Union State. Brazil, 2006

\begin{tabular}{|c|c|c|c|c|c|c|}
\hline Região e Unidade da Federação & População & $\begin{array}{l}\text { Inter } \\
\text { SUS }\end{array}$ & $\begin{array}{l}\text { Ind. } \\
\text { SUS }\end{array}$ & $\begin{array}{c}\text { Ind. } \\
\text { SUS e não SUS }\end{array}$ & Tx padr. $^{4}$ & $\operatorname{Pos}^{5}$ \\
\hline Brasil & 186.770 .560 & 6,1 & 5,0 & 5,6 & 5,6 & \\
\hline Norte & 15.022 .064 & 6,7 & 6,0 & 6,1 & 6,4 & \\
\hline Acre & 676.625 & 7,7 & 6,6 & 6,9 & 7,2 & (1) \\
\hline Amapá & 615.714 & 5,7 & 5,4 & 5,4 & 6,0 & (8) \\
\hline Amazonas & 3.321 .055 & 5,4 & 4,9 & 4,9 & 5,2 & (12) \\
\hline Pará & 7.110 .467 & 7,3 & 6,7 & 6,8 & 7,1 & (2) \\
\hline Rondônia & 1.562 .418 & 6,1 & 5,5 & 5,6 & 5,9 & (9) \\
\hline Roraima & 403.344 & 5,2 & 4,8 & 5,2 & 5,7 & (11) \\
\hline Tocantins & 1.332 .441 & 7,5 & 6,3 & 6,4 & 6,6 & (5) \\
\hline Nordeste & 51.609 .020 & 6,2 & 5,4 & 5,5 & 5,6 & \\
\hline Alagoas & 3.050 .650 & 6,5 & 5,7 & 5,7 & 5,9 & (9) \\
\hline Bahia & 13.950 .147 & 6,4 & 5,5 & 5,6 & 5,7 & (11) \\
\hline Ceará & 8.217 .083 & 6,3 & 5,4 & 5,6 & 5,7 & (11) \\
\hline Maranhão & 6.184 .538 & 6,1 & 5,5 & 5,7 & 6,0 & (8) \\
\hline Paraíba & 3.623 .218 & 6,5 & 5,7 & 5,8 & 5,8 & (10) \\
\hline Pernambuco & 8.502 .604 & 6,0 & 5,2 & 5,2 & 5,2 & (12) \\
\hline Piauí & 3.036 .285 & 7,0 & 6,3 & 6,5 & 6,7 & (4) \\
\hline Rio Grande do Norte & 3.043 .759 & 5,4 & 4,8 & 4,8 & 4,9 & (14) \\
\hline Sergipe & 2.000 .736 & 5,4 & 4,6 & 4,7 & 4,8 & (15) \\
\hline Centro-Oeste & 13.269 .520 & 6,9 & 5,8 & 6,2 & 6,4 & \\
\hline Goiás e Distrito Federal & 8.114 .539 & 6,9 & 5,7 & 6,1 & 6,3 & (6) \\
\hline Mato Grosso & 2.856 .999 & 6,4 & 5,5 & 5,9 & 6,2 & (7) \\
\hline Mato Grosso do Sul & 2.297 .982 & 7,7 & 6,4 & 6,8 & 6,9 & (3) \\
\hline Sudeste & 79.561 .095 & 5,5 & 4,3 & 5,4 & 5,2 & \\
\hline Espírito Santo & 3.464 .284 & 5,4 & 4,7 & 5,1 & 5,1 & (13) \\
\hline Minas Gerais & 19.479 .355 & 6,1 & 4,9 & 5,3 & 5,2 & (12) \\
\hline Rio de Janeiro & 15.561 .721 & 4,5 & 3,9 & 4,0 & 3,8 & (16) \\
\hline São Paulo & 41.055 .735 & 5,5 & 4,2 & 5,9 & 5,8 & (10) \\
\hline Sul & 27.308 .861 & 6,8 & 5,2 & 6,2 & 6,1 & \\
\hline Paraná & 10.387 .378 & 7,0 & 5,4 & 5,9 & 5,9 & (9) \\
\hline Rio Grande do Sul & 10.963 .219 & 6,8 & 5,1 & 6,5 & 6,2 & (7) \\
\hline Santa Catarina & 5.958 .264 & 6,3 & 4,9 & 6,0 & 6,0 & (8) \\
\hline
\end{tabular}

Fonte: MS/Datasus - Sistema de Informação Hospitalar (SIH) e Comunicação de Internação Hospitalar (CIH) competências 2006 e 2007.

${ }^{1}$ internação SUS com base nas autorizações de internações hospitalares do tipo $1 ;{ }^{2}$ indivíduos com ao menos uma internação pelo SUS; ${ }^{3}$ indivíduos internados pelo SUS e por financiamento não SUS; ${ }^{4}$ taxa SUS e não SUS padronizada pela faixa etária da população; ${ }^{5}$ em ordem decrescente da taxa padronizada Source: MS/Datasus - Hospital Information System (HIS) and Communication Hospital (CIH) 2006 and 2007.

'SUS Admissions based in the Hospital Admissions Authorization 1; ${ }^{2}$ individuals with at least one SUS admission; ${ }^{3}$ individuals admitted by SUS and by non SUS financing; ${ }^{4}$ SUS and non SUS rate standardized by population age $;$ in the standardized rate decrescent order

permanência de recém-nascido. Vale destacar que as internações cujo motivo de cobrança ou de comunicação indicava internação não finalizada, foram consideradas como casos cujo paciente permaneceu internado e representaram $3,4 \%$ no país. Esse valor refere-se às internações para as quais já havia sido apresentada ao menos uma cobrança parcial. As proporções de altas são maiores e das transferências e óbitos menores nas internações não SUS do que no SUS (Tabela 3).

Quanto ao diagnóstico principal, analisado por capítulo da Classificação Internacional de Doenças - $10^{\mathrm{a}}$ revisão (CID-10), observa-se que o capítulo referente 


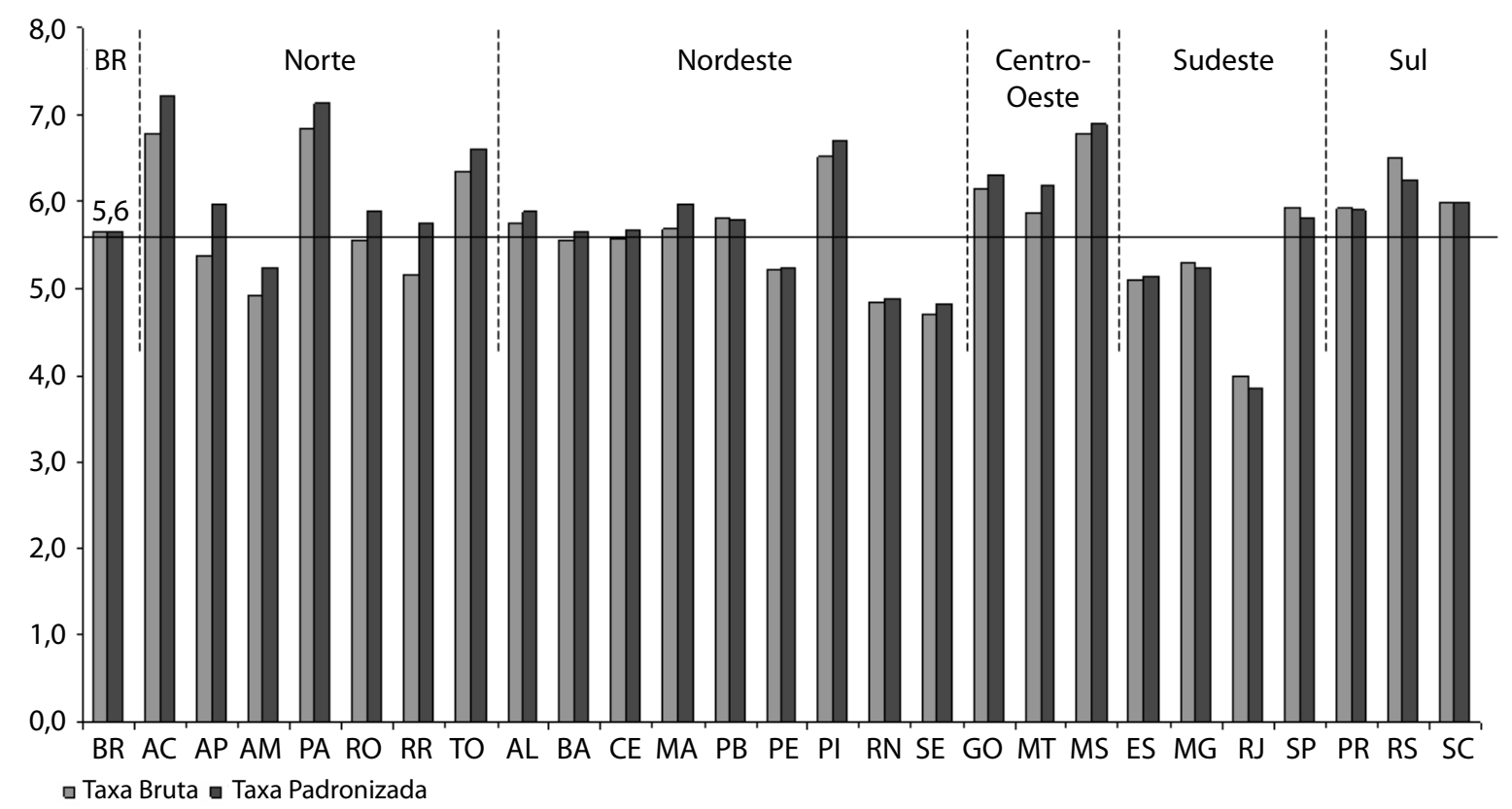

Fonte: MS/Datasus - Sistema de Informação Hospitalar (SIH) e Comunicação de Internação Hospitalar (CIH) competências 2006 e 2007.

*taxa padronizada, método direto, nas faixas etárias da população: menor de 1 ano, 1 a 4 anos, 5 a 9 anos, 10 a 19 anos, 20 a 59 anos e 60 anos e mais - Brasil, 2006 Source: MS/Datasus - Hospital Information System (HIS) and Communication Hospital (CIH) 2006 and 2007.

*standardized rate, the direct method, the population in the age groups: under 1 year, 1-4 years, 5-9 years, 10-19 years, 20-59 years and 60 years and over - Brazil, 2006

Figura 1. Internação hospitalar por 100 habitantes* residentes na Unidade Federada. Brasil, 2006

Figure 1. Hospital admissions per 100 habitants* of Union States. Brazil, 2006

Tabela 3. Proporção de internações que utilizaram a unidade de terapia intensiva (UTI) segundo a faixa etária, o sexo, o motivo de saída, por fonte de financiamento (SUS ou não SUS). Brasil, 2006

Table 3. Proportion of admissions with intensive care unit (ICU) use by age group, sex, reason for discharge and type of payment (SUS or non-SUS). Brazil, 2006

\begin{tabular}{lccc}
\hline Características das internações & SUS & Não SUS & TOTAL \\
\hline Internação & 10.898 .197 & 1.493 .793 & 12.391 .990 \\
Internação com UTI & 458.821 & 64.876 & 523.697 \\
\% Internação com UTI & 4,2 & 4,3 & 4,2 \\
Faixa etária & & & \\
$<1$ ano & 17,6 & 8,7 & 16,5 \\
1 a 4 anos & 3,0 & 2,1 & 2,9 \\
5 a 9 anos & 1,8 & 1,1 & 1,7 \\
10 a 19 anos & 3,9 & 2,1 & 3,7 \\
20 a 59 anos & 36,5 & 30,6 & 35,8 \\
60 anos e mais & 37,2 & 55,4 & 39,4 \\
Sexo & & & \\
Masculino & 55,7 & 51,8 & 55,2 \\
Feminino & 44,3 & 48,2 & 44,8 \\
Motivo de Saída & & & \\
Alta & 68,3 & 76,7 & 69,4 \\
Transferência & 4,8 & 2,8 & 2,5 \\
Óbito & 23,2 & 18,2 & 0,1 \\
Permanência recém-nascido & 0,1 & 0,0 & 3,4 \\
Internado & 3,6 & 2,3 & \\
\hline
\end{tabular}

Fonte: MS/Datasus - Sistema de Informação Hospitalar (SIH) e Comunicação de Internação Hospitalar (CIH) competências 2006 e 2007. Source: MS/Datasus - Hospital Information System (HIS) and Communication Hospital (CIH) 2006 and 2007. 
Tabela 4. Proporção de internações e posição por capítulo da Classificação Internacional de Doenças (CID) e fonte de financiamento. Brasil, 2006

Table 4. Proportion of admissions according to International Classification of Diseases (ICD) chapter and type of payment. Brazil, 2006

\begin{tabular}{|c|c|c|c|c|c|c|}
\hline \multirow{2}{*}{ Capítulo da CID } & \multicolumn{2}{|c|}{ SUS } & \multicolumn{2}{|c|}{ Não SUS } & \multicolumn{2}{|c|}{ TOTAL } \\
\hline & \multicolumn{2}{|c|}{ \% (Posição) } & \multicolumn{2}{|c|}{ \% (Posição) } & \multicolumn{2}{|c|}{ \% (Posição) } \\
\hline Internação & \multicolumn{2}{|c|}{ 10.898.197 } & \multicolumn{2}{|c|}{1.493 .793} & \multicolumn{2}{|c|}{12.391 .990} \\
\hline$\%$ & 87,9 & & 12,1 & & 100,0 & \\
\hline I. Algumas doenças infecciosas e parasitárias & 9,05 & (4) & 5,72 & (9) & 8,65 & (5) \\
\hline II. Neoplasias (tumores) & 5,26 & $(8)$ & 6,01 & $(8)$ & 5,35 & (8) \\
\hline III. Doenças do sangue e transtornos imunitários & 0,68 & $(18)$ & 0,56 & $(17)$ & 0,66 & (18) \\
\hline IV. Doenças endócrinas e outras & 2,58 & $(10)$ & 3,85 & $(11)$ & 2,73 & $(10)$ \\
\hline V. Transtornos mentais e comportamentais & 3,03 & (9) & 1,78 & (14) & 2,88 & (9) \\
\hline VI. Doenças do sistema nervoso & 1,50 & $(13)$ & 2,11 & $(13)$ & 1,57 & (14) \\
\hline VII. Doenças do olho e anexos & 0,36 & (19) & 0,29 & $(20)$ & 0,35 & (19) \\
\hline VIII. Doenças do ouvido e apófise mastóide & 0,14 & $(20)$ & 0,43 & (19) & 0,17 & $(20)$ \\
\hline IX. Doenças do aparelho circulatório & 10,09 & (3) & 12,03 & $(2)$ & 10,33 & (3) \\
\hline X. Doenças do aparelho respiratório & 13,86 & $(2)$ & 10,95 & (3) & 13,51 & (2) \\
\hline XI. Doenças do aparelho digestivo & 8,60 & $(5)$ & 10,67 & $(4)$ & 8,85 & $(4)$ \\
\hline XII. Doenças da pele e tecido subcutâneo & 1,26 & (15) & 1,05 & (16) & 1,24 & (16) \\
\hline XIII. Doenças do sistema osteomuscular e do tecido conjuntivo & 2,25 & $(11)$ & 4,52 & $(10)$ & 2,53 & $(11)$ \\
\hline XIV. Doenças do aparelho geniturinário & 6,53 & (7) & 8,46 & $(5)$ & 6,76 & (6) \\
\hline XV. Gravidez, parto e puerpério & 22,96 & (1) & 13,96 & (1) & 21,87 & (1) \\
\hline XVI. Algumas afecções originadas no período perinatal & 1,89 & $(12)$ & 1,12 & $(15)$ & 1,79 & $(13)$ \\
\hline $\begin{array}{l}\text { XVII. Malformações congênitas, deformidades e anomalias } \\
\text { cromossômicas }\end{array}$ & 0,69 & $(17)$ & 0,54 & $(18)$ & 0,67 & (17) \\
\hline $\begin{array}{l}\text { XVIII. Sintomas, sinais e achados anormais de exames clínicos e } \\
\text { de laboratórios, não classificados em outra parte }\end{array}$ & 1,29 & (14) & 6,65 & $(6)$ & 1,94 & (12) \\
\hline $\begin{array}{l}\text { XIX. Lesões, envenenamentos e } \\
\text { algumas outras consequências de causas externas }\end{array}$ & 6,80 & (6) & 6,29 & $(7)$ & 6,74 & (7) \\
\hline XX. Causas externas & 0,01 & $(21)$ & 0,18 & $(21)$ & 0,03 & (21) \\
\hline XXI. Contatos com serviços & 1,17 & (16) & 2,83 & (12) & 1,37 & (15) \\
\hline
\end{tabular}

Fonte: MS/Datasus - Sistema de Informação Hospitalar (SIH) e Comunicação de Internação Hospitalar (CIH) competências 2006 e 2007. Source: MS/Datasus - Hospital Information System (HIS) and Communication Hospital (CIH) 2006 and 2007.

à gravidez, parto e puerpério $(\mathrm{XV})$ inclui o maior número de internações no Brasil $(21,9 \%)$ (Tabela 4). Os capítulos das doenças do aparelho respiratório (X) e das doenças do aparelho circulatório (IX) ocupam a segunda e a terceira posição. Esses três capítulos, juntamente com os capítulos referentes às doenças do aparelho digestivo (XI), doenças infecciosas e parasitárias (I) e doenças do aparelho geniturinário (XIV), concentram $70 \%$ dos principais motivos de internações ocorridas no país em 2006.

A Tabela 4 mostra diferença na distribuição das causas das internações não SUS quando comparadas às das internações SUS. Nesse sentido, destaca-se nas internações não SUS a menor participação dos capítulos das doenças infecciosas e parasitárias, transtornos mentais, doenças do aparelho respiratório e gravidez, parto e puerpério e a maior participação das doenças do aparelho digestivo, doenças do sistema osteomuscular e tecido conjuntivo, doenças do aparelho geniturinário e, principalmente, do capítulo sintomas, sinais e achados (XVIII) (6,7\% não SUS versus 1,3\% SUS). O valor elevado para internações classificadas nesse capítulo, bem como no de contatos com serviços de saúde (XXI), ainda que em menor intensidade, sinaliza a maior dificuldade ainda existente no preenchimento da $\mathrm{CIH}$. 
Tabela 5. Proporção de internações e posição segundo os procedimentos realizados mais frequentes e fonte de financiamento. Brasil, 2006

Table 5. Proportion of admissions according to frequent procedures and type of payment. Brazil, 2006

\begin{tabular}{|c|c|c|c|c|c|c|}
\hline \multirow{2}{*}{ Procedimento } & \multicolumn{2}{|c|}{ SUS } & \multicolumn{2}{|c|}{ Não SUS } & \multicolumn{2}{|c|}{ TOTAL } \\
\hline & \multicolumn{2}{|c|}{ \% (Posição) } & \multicolumn{2}{|c|}{ \% (Posição) } & \multicolumn{2}{|c|}{ \% (Posição) } \\
\hline Internações & \multicolumn{2}{|c|}{10.898 .197} & \multicolumn{2}{|c|}{1.493 .793} & \multicolumn{2}{|c|}{12.391 .990} \\
\hline Parto normal & 13,66 & $(1)$ & 1,97 & (6) & 12,25 & $(1)$ \\
\hline Pneumonia & 7,27 & $(2)$ & 3,73 & (4) & 6,84 & $(2)$ \\
\hline Cesariana & 5,95 & (3) & 9,47 & (2) & 6,37 & (3) \\
\hline Entero-infecções & 5,20 & (4) & 3,39 & (5) & 4,98 & (4) \\
\hline Diagnóstico e/ou primeiro atendimento & 2,18 & (8) & 10,59 & (1) & 3,20 & (5) \\
\hline Insuficiência cardíaca & 2,87 & (5) & 1,85 & (7) & 2,75 & (6) \\
\hline Tratamento em psiquiatria & 2,83 & (6) & 1,43 & (8) & 2,67 & (7) \\
\hline Crise asmática & 2,48 & (7) & 0,83 & $(15)$ & 2,28 & (8) \\
\hline Curetagem pós aborto & 1,99 & (9) & 0,74 & $(17)$ & 1,84 & (9) \\
\hline $\begin{array}{l}\text { Tratamento clínico por acidente vascular cerebral isquêmico ou } \\
\text { hemorrágico agudo }\end{array}$ & 1,54 & $(10)$ & 1,30 & $(10)$ & 1,51 & (10) \\
\hline Pielonefrite & 1,50 & $(11)$ & 0,83 & $(15)$ & 1,42 & (11) \\
\hline Doença pulmonar obstrutiva crônica & 1,44 & $(12)$ & 0,89 & $(13)$ & 1,37 & (12) \\
\hline Hemorragia inguinal & 1,36 & (13) & 1,09 & $(11)$ & 1,33 & (13) \\
\hline Crise hipertensiva & 1,18 & $(14)$ & 1,41 & (9) & 1,21 & (14) \\
\hline Colecistectomia & 1,09 & $(16)$ & 1,41 & (9) & 1,13 & (15) \\
\hline Diabetes sacarino & 1,13 & (15) & 0,71 & $(18)$ & 1,08 & (16) \\
\hline Intercorrências clínicas de paciente oncológico & 1,08 & $(17)$ & 0,80 & $(16)$ & 1,04 & (17) \\
\hline Procedimento não constante em SIH/SUS & 0,00 & $(20)$ & 6,82 & (3) & 0,82 & (18) \\
\hline Insuficiência coronariana aguda & 0,76 & (19) & 1,02 & $(12)$ & 0,79 & (19) \\
\hline Apendicectomia & 0,77 & $(18)$ & 0,87 & $(14)$ & 0,78 & $(20)$ \\
\hline
\end{tabular}

Fonte: MS/Datasus - Sistema de Informação Hospitalar (SIH) e Comunicação de Internação Hospitalar (CIH) competências 2006 e 2007. Source: MS/Datasus - Hospital Information System (HIS) and Communication Hospital (CIH) 2006 and 2007.

Na Tabela 5, que apresenta os principais procedimentos realizados nas internações SUS e não SUS, observa-se que o perfil dos 20 procedimentos mais frequentes no SUS, acompanha a distribuição dos procedimentos nas internações em geral, e que existem diferenças importantes com o observado no não SUS. O parto normal ocupa a primeira posição no geral e no SUS, mas passa para a sexta posição entre as internações não SUS; entre os procedimentos não SUS, a cesariana ocupa a segunda posição e, no SUS, a terceira. Entre os não SUS, destaca-se o procedimento para diagnóstico ou primeiro atendimento na primeira posição, enquanto que no SUS este ficou na oitava posição, novamente uma indicação de menor qualidade no preenchimento da CIH. Nesse sentido, também se destaca o expressivo volume do item "procedimentos não constantes na tabela do SIH/SUS", a terceira posição no não SUS.

\section{Discussão}

O uso de dados administrativos em avaliações de saúde é usual em razão do custo da coleta no prontuário do paciente e por estarem disponíveis em escala que possibilita estudos em diversas perspectivas ${ }^{23}$. Dos três sistemas fontes aqui empregados, o SIH tem sido utilizado em diversas pesquisas $^{3,12,13,18,19}$, o CNES só recentemente passou a ser utilizado em estudos ${ }^{24}$ e a $\mathrm{CIH}$ ainda tem uso incipiente na análise da assistência hospitalar.

Considerando-se o volume de unidades e registros que compuseram a base de dados inicial da pesquisa, destaca-se a coerência na série histórica, com envio mensal dos dados do SUS pelos hospitais. Os dados 
não SUS contribuíram com $12,1 \%$ dos registros e têm abrangência nacional, permitindo uma primeira aproximação da assistência hospitalar não SUS.

O uso de dados administrativos implicou a reorganização dos mesmos, efetuada na composição dos dados do atendimento a partir das cobranças. A complexidade dessa tarefa esteve também relacionada à compreensão dos conceitos de pagamento adotados no SUS, e consequente a organização dos dados de pagamentos em dados de internação. Os achados indicaram inconsistências no preenchimento dos campos de nome, procedimento, duplicidade de registros e sobreposição parcial no período de atendimento em 2,4\% dos registros iniciais, proporção esta relativamente baixa que não compromete o estudo.

Para traçar o perfil de internações de 2006, os dados disponíveis foram analisados por local de residência, ainda que a localização do indivíduo internado tenha sido por ocorrência, por terem sido considerados como uma boa proxy para analisar a assistência hospitalar nesses anos. As diferentes metodologias aplicadas para o cálculo da taxa de internação por 100 residentes e a inclusão de dados não SUS, representando um aprimoramento do cálculo pelo método usual, não produziu resultado tão diferente justificando a sua utilização na gestão. Interessante observar que, considerando-se haver sub-registro da CIH, a taxa de internação final obtida foi semelhante à encontrada na PNAD 20037, porém inferior à encontrada na PNAD 2008. A inclusão dos dados não SUS possibilitou uma primeira quantificação da sua participação nas áreas mais populosas com acesso diferenciado a internações por outras fontes de financiamento que não o SUS, contribuindo para o conhecimento hoje disponível sobre a assistência hospitalar no país.

Quanto às taxas de internação por UF, o SUS, nas formas de cálculo utilizadas, apresenta o perfil semelhante ao da taxa total de internação, dado o predomínio das internações SUS no total de internações. As taxas obtidas para o não SUS contribuem para a melhor compreensão da assistência hospitalar à luz da oferta de serviços, SUS e não SUS, e utilização de planos de saúde. As taxas observadas mostram-se, em geral, maiores nos estados com nível socioeconômico mais elevado e menores nos estados com menor nível socioeconômico. Os valores elevados em alguns estados da Região Norte e o valor muito baixo no Rio de Janeiro exigem uma análise cuidadosa adicional, para a sua compreensão.

A comparação da taxa nacional de internação de 56 por 1.000 habitantes com outros países deve ser feita com muito cuidado, contudo a taxa de internação média dos países da OCDE é de 158 por 1.000 habitantes, apresentando como valores extremos a taxa de 278 por 1.000 habitantes, na Áustria, e 55 por 1.000, no México $^{25}$. É grande a variabilidade entre os países e não há uma relação direta entre renda per capita e as taxas de internação, observando-se que países desenvolvidos com sistemas de saúde com cobertura universal como a Holanda (109 por 1.000) e Canadá (84 por 1.000 habitantes) apresentam valores relativamente mais baixos ${ }^{25}$ do que países com renda semelhante e sistemas de saúde com acesso populacional diferenciado, indicando a influência do tipo de sistema de saúde, do acesso aos serviços e da organização da assistência sobre a taxa de internação.

A proporção de internações com utilização de UTI não mostrou diferença entre SUS e não SUS. Mas no não SUS, a proporção de internações de indivíduos com mais de 60 anos é ainda mais significativa do que no SUS, observando-se o contrário na participação dos indivíduos menores de 1 ano. Quanto à distribuição das internações por capítulos da CID, foram observadas diferenças importantes entre o SUS e não SUS. A identificação dessas diferenças na assistência prestada no SUS e não SUS permite a definição de pesquisas complementares necessárias para a sua compreensão.

As internações não SUS apresentam uma participação, ainda que pequena, de internações classificadas no capítulo das causas externas, sendo o recomendado para a 
codificação do diagnóstico principal a utilização de categorias do capítulo lesões e envenenamentos, onde o capítulo de causas externas seria empregado para o campo do diagnóstico secundário, salvo situações excepcionais. No financiamento SUS, essa situação também está presente, porém em proporção bem menor. Essas diferenças nas proporções observadas para as internações por fonte de financiamento devem ser interpretadas com cuidado por serem resultantes de combinações complexas entre contextos muito variáveis, como perfil epidemiológico da população, capacidade instalada, acesso aos serviços e qualidade da informação.

Ainda que haja similaridade entre os cinco principais procedimentos realizados nas duas fontes de financiamento, existem diferenças importantes de difícil interpretação entre os principais procedimentos, há uma menor proporção relativa de internações para tratamento de crise asmática, curetagem pós-aborto, tratamento em psiquiatria e diabetes, e maior proporção de internações por colecistectomia e insuficiência coronariana fora do SUS.

Por empregar dados administrativos, esse estudo apresenta certo grau de limitação para a análise das internações hospita$\operatorname{lares}^{23}$. Outra limitação possível é a subnotificação decorrente dos tetos financeiros para pagamento da internação no SUS que podem ter restringido o envio de dados à base nacional. Além disso, a subnotificação da CIH foi um fator limitante nas análises e a inexistência de uma tabela padronizada de procedimentos médico-hospitalares limitou a análise por esta variável. Embora o sub-registro seja uma limitação ao uso da $\mathrm{CIH}$, a hipótese de que não há tendência de perda de registros para um determinado sexo ou grupo etário, ou mesmo para um conjunto específico de causas de internação, reforça a relevância dos resultados apresentados para conhecimento da distribuição demográfica e do perfil de morbidades das internações. O sub-registro afetaria mais fortemente as estimativas das taxas de internação; contudo pode-se supor que o perfil esteja próximo do obtido, caso todos os registros estivessem sendo considerados. A utilização de método probabilístico para identificação do indivíduo, com parâmetros conservadores, tal como a exata data de nascimento para associação dos registros, a fim de evitar a inclusão de falsos positivos, também pode subestimar os resultados encontrados, por possibilitar a subidentificação dos indivíduos. Por outro lado, garante maior confiança na estratégia aqui adotada para a compatibilização dos sistemas de informação. Tais artifícios seriam desnecessários se medidas como a adoção de identificador único do indivíduo e do atendimento fossem, entre outras implantadas.

Em conclusão, os dados das internações não financiadas pelo SUS coletados pela CIH agregam informações importantes para a análise da assistência hospitalar no país. Os dados administrativos do SUS são válidos para análises de internações e os algoritmos de composição dos dados de internação, a partir da cobrança, aprimoram a análise do Sistema de Serviços Hospitalares no Brasil.

\section{Referências}

1. Travassos C, Viacava F, Fernandes C, Almeida CM. Desigualdades geográficas e sociais na utilização de serviços de saúde no Brasil. Ciênc Saúde Coletiva. 2000;5(1):133-49.

2. Travassos C, Martins M. Uma revisão sobre os conceitos de acesso e utilização de serviços de saúde. Cad Saúde Pública. 2004;20(Suppl 2):S190-8.
3. Oliveira EXG, Travassos C, Carvalho MS. Acesso à internação hospitalar nos municípios brasileiros em 2000: territórios do Sistema Único de Saúde. Cad Saúde Pública. 2004;20(Suppl 2):S298-309.

4. Travassos C, Novaes HMD. Investigação e avaliação em serviços de saúde [editorial]. Cad Saúde Pública. 2004;20(Suppl 2):S144-5. 
5. Castro MSM, Travassos C, Carvalho MS. Efeito da oferta de serviços de saúde no uso de internações hospitalares no Brasil. Rev Saúde Pública. 2005;39(2):277-84.

6. Ribeiro JM. Desenvolvimento do SUS e racionamento de serviços hospitalares. Ciênc Saúde Coletiva. 2009;14(3):771-82.

7. Castro MSM. Desigualdades sociais no uso de internações hospitalares no Brasil: o que mudou entre 1998 e 2003. Ciênc Saúde Coletiva. 2006;11(4):987-98.

8. Porto SM, Santos IS, Ugá MAD. A utilização de serviços de saúde por sistema de financiamento. Ciênc Saúde Coletiva. 2006;11(4):895-910.

9. Ribeiro MCSA, Barata RB, Almeida MF, Silva ZP. Perfil sociodemográfico e padrão e utilização de serviços de saúde para usuários do SUS-PNAD 2003. Ciênc Saúde Coletiva. 2006;11(4):1011-22.

10. Brasil. Ministério do Orçamento e Gestão. Instituto Brasileiro de Geografia e Estatística. Pesquisa Nacional por Amostra de Domicílios: Um Panorama da Saúde no Brasil: acesso e utilização de Serviços de Saúde, Condições de Saúde e Fatores de Risco e Proteção à Saúde 2008. Rio de Janeiro: IBGE; 2009.

11. Brasil. Ministério da Saúde. Portaria no 1.722 de 22 de setembro de 2005. Altera a estrutura da Comunicação de Internação Hospitalar e dá outras providências. Diário Oficial da União; 2005Set 23; Seção 1:43.

12. Oliveira EXG, Carvalho MS, Travassos C. Territórios do Sistema Único de Saúde: mapeamento das redes de atenção hospitalar.Cad Saúde Pública. 2004;20(2):386-402.

13. Amaral ACS, Coeli CM, Costa MCE, Cardoso VS, Toledo ALA, Fernandes, CR. Perfil de morbidade e de mortalidade de pacientes idosos hospitalizados. Cad Saúde Pública. 2004;20(6):1617-26.

14. Ray WA. Policy and program analysis using administrative database. Ann Intern Med. 1997;127(8 Pt 2):712-8.

15. Cooper GS, Schultz L, Simpkins J, Lafata JE. The utility of administrative data for measuring adherence to cancer surveillance care guidelines. Med Care. 2007;45(1):66-72.

16. Scott I, Youlden D, Coory M. Are diagnosis specific outcome indicators based on administrative data useful in assessing quality of hospital care? Qual Saf Health Care. 2004;13(1):32-9.

17. Urbach DR, Baxter NN. Does it matter what a hospital is "high volume" for? Specificity of hospital volumeoutcome associations for surgical procedures: analysis of administrative data. Qual Saf Health Care. 2004;13(5):379-83.

18. Pinheiro RS, Vieira RA, Coeli CM, Vidal EIO, Camargo Júnior KR. Utilização do SIH-SUS e do SIM para o cálculo da mortalidade hospitalar em 30 dias para as internações de pacientes com fratura proximal do fêmur. Cad Saúde Colet (Rio J). 2006;14(2):337-44.

19. Teixeira CLS, Bloch KV, Pinheiro RS, Klein CH. Concordâncias entre causas de óbito nas declarações de óbito e diagnósticos nas autorizações de internação hospitalar em regiões do Estado do Rio de Janeiro, em 1998, usando relacionamento probabilístico de dados. Cad Saúde Colet (Rio J) 2006;14(2):405-10.

20. Camargo Júnior KR, Coeli CM. Recklink: aplicativo para o relacionamento de bases de dados implementando o método probabilistic record linkage. Cad Saúde Pública. 2000;16(2):439-47.

21. Coeli CM, Camargo Júnior KR. Avaliação de diferentes estratégias de blocagem no relacionamento probabilístico de registros. Rev Bras Epidemiol. 2002;5(2):185-96.

22. Rede Interagencial de Informações para a Saúde. Indicadores básicos para a saúde no Brasil: conceitos e aplicações. Opas: Brasília; 2008.

23. Iezzoni LI. Assessing quality using administrative data. Ann Intern Med. 1997;127(8 Pt 2):666-74.

24. Carvalho CA. Cadastro Nacional de Estabelecimentos de Saúde - seu desenvolvimento e implantação e uma proposta para sua atualização/manutenção. [dissertação]. Rio de Janeiro: Escola Nacional de Saúde Pública. Fundação Osvaldo Cruz. Ministério da Saúde; 2004.

25. OCDE Health Data 2009. [cited 2011 Sept 06]. Available from: <http://dx.doi.org/10.1787/718432575088>.

Recebido em: 12/03/2010 Versão final apresentada em: 13/06/2011 Aprovado em: 25/08/2011 